\title{
¿ेPara qué sirve la escuela? Reflexiones sociológicas en tiempos de pandemia global
}

\author{
What Are Schools For? Sociological Reflections \\ in Times of Global Pandemic
}

\section{Aina Tarabini ${ }^{1}$}

\begin{abstract}
Resumen
La pandemia global generada por el COVID-19 y las medidas de confinamiento obligatorio de la población que de ella se derivan suponen retos sin precedentes para los sistemas educativos a nivel global. El cierre generalizado de todos los centros educativos a nivel presencial pone de relieve una pregunta clave: ¿Para qué sirve su escuela? El objetivo del artículo es reflexionar sobre esta cuestión desde una mirada sociológica y, en particular, desde un enfoque de la justicia educativa y social. Se argumenta que la función principal de la escuela como institución especializada debe ser la transmisión y adquisición de conocimientos profundos, relevantes y con sentido para todos y todas las estudiantes. Asimismo, se establecen dos requisititos para garantizar el ejercicio de este rol: la presencia física como base para poder desarrollar una interacción plena; y el rol de los y las docentes como acompañantes. En conjunto, el artículo presenta una reflexión no solo sobre los impactos de la situación actual sobre las funciones de la escuela, sino también sobre el sentido de la institución escolar en pleno siglo xxi, representando un aporte clave dentro de los debates de la sociología de la educación.
\end{abstract}

\section{Palabras clave}

Sociología de la educación, justicia social, funciones escuela, aprendizaje, presencia, docentes.

\section{Abstract}

The global pandemic generated by COVID-19 and the consequent mandatory confinement measures of the population entail unprecedented challenges for the education systems elsewhere. The closing of the schools and of the face-to-face classes highlights a critical question: What is the school for? The objective of the paper is to reflect on this question from a sociological perspective and, in particular, from an educational and social justice perspective. It is argued that the main function of the school as a specialized institution should be the transmission and acquisition of powerful, relevant and meaningful knowledge for all students. Likewise, two requirements are established to guarantee the exercise of this role: the physical presence as the basis for developing full interaction; and the role of teachers to simultaneously ensure students' learning and wellbeing. Overall, the paper provides a reflection not only on the impacts of the current situation on the functions of the school, but also on the meaning of the educational institution in the xxI century, representing a key contribution within the debates of the sociology of education.

\section{Keywords}

Sociology of education, social justice, purposes of schooling, learning, presence, teachers.

\section{Cómo citar/Citation}

Tarabini, Aina (2020). ¿Para qué sirve la escuela? Reflexiones sociológicas en tiempos de pandemia global. Revista de Sociología de la Educación-RASE, 13 (2) Especial, COVID-19, 145-155. https://doi.org/10.7203/RASE.13.2.17135. 


\section{Introducción}

La pandemia global generada por el COVID-19 y las medidas de confinamiento obligatorio de la población que de ella se derivan suponen retos sin precedentes para los sistemas educativos a nivel global. El cierre generalizado de todos los centros educativos a nivel presencial pone sobre la mesa una pregunta fundamental: ¿Para qué sirve la escuela? ¿Cuáles son y deben ser sus funciones en pleno siglo xxi? Esta pregunta se encuentra en el núcleo de los debates de la Sociología de la Educación desde su institucionalización como disciplina en los años 50 del siglo pasado pero hoy, en un momento en que todo lo que se daba por hecho se torna incierto, cobra su máximo sentido.

El artículo que se presenta a continuación tiene por objetivo reflexionar sobre esta cuestión desde un enfoque de la justicia educativa y social (Lynch y Baker, 2005). Para ello, en el primer apartado, se hace un breve repaso a las funciones de los sistemas educativos señaladas por la Sociología de la Educación, así como a sus repercusiones y significados en términos de desigualdad social. En el segundo, se discute sobre los impactos de la situación actual sobre el ejercicio de dichas funciones y se reflexiona sobre sus múltiples repercusiones en términos de oportunidades educativas. En el tercer apartado se presenta el eje central que, a mi entender, debe marcar el rol de la escuela como institución especializada: la transmisión y adquisición de conocimientos profundos, relevantes y con sentido para todos y todas las estudiantes. Asimismo, se establecen dos requisititos para garantizar el ejercicio de este rol: la presencia física como base para poder desarrollar una interacción plena, clave en los procesos de transmisión cultural; y el rol de los y las docentes como acompañantes en el marco de una escuela que actúa simultáneamente como comunidad de aprendizaje y de cuidados. En el último apartado se reflexiona, a modo de conclusión, sobre el sentido de la escuela como institución y sobre las condiciones para poder garantizar su función como instrumento de igualación social.

\section{Sobre las funciones (desiguales) de los sistemas educativos}

Desde la creación de los sistemas educativos modernos, éstos han tenido dos grandes funciones complementarias junto con la de custodia: la formación de las nuevas generaciones para ejercer una profesión y su preparación para desarrollar la vida en sociedad. Desde las corrientes funcionalistas (Parsons, 1990) hasta las teorías marxistas (Althusser, 1985), pasando por la teoría de la reproducción (Bourdieu y Passeron, 1970; Bernstein, 1985a, 1985b), la teoría de la correspondencia (Bowles y Gintis, 1976) o la denominada en su momento 'nueva sociología de la educación' (Young, 1971), todas coinciden en señalar que socialización y selección son las dos grandes funciones de los sistemas educativos, desde la creación de las sociedades capitalistas industriales (Bonal, 1998). Funciones que han sido concebidas, valoradas y explicadas de forma diferente por diferentes corrientes teóricas pero nunca cuestionadas por sí mismas.

La escuela, como institución especializada ${ }^{2}$, se encarga de transmitir conocimientos, habilidades y destrezas a la vez que forma en actitudes, disposiciones y carácteres; forma sujetos, crea identidades. Se trata, de hecho, de una institución privilegiada para la creación de identidades sociales (Reay, 2010). Sin duda, existen otros agentes e instituciones sociales encargados de la transmisión de conocimientos y de la socialización de niños, niñas y jóvenes. La familia, evidentemente, como también las entidades de ocio educativo y, de forma creciente, las tecnologías de la información y la comunicación (TIC). Sin embargo, la escuela representa un contexto institucional con intencionalidad educativa explícita que no puede ser

2 En este artículo se utiliza la noción de institución para referirse a la función de instruir y socializar. Siguiendo a Dubet (2010: 16) entiendo que 'una institución se define por su capacidad de promover un orden simbólico y formar un tipo de sujeto amoldado a cierto orden, en definitiva, de instituirle'. 
sustituida de forma mecánica por otros agentes sociales. Y ello es así, como argumentaré en los siguientes apartados, por diversos motivos. Uno de los principales, a mi entender, es la función de la escuela pública, común, obligatoria y gratuita como instrumento de igualación social (Gimeno Sacristán, 2000). Función, que si bien se realiza de forma incompleta y sesgada por las múltiples formas de exclusión y desigualdad que atraviesan nuestros sistemas educativos (Tarabini et al., 2017), solo puede desarrollar la escuela como institución social. De ahí su especificidad. Volveré sobre esta cuestión en el siguiente apartado. Antes, sin embargo, quiero apuntar brevemente la forma a partir de la cual las funciones de la institución escolar se ejercen de forma desigual, poniendo en cuestión su rol para garantizar la igualdad y la justicia social. Es precisamente esta desigualdad de oportunidades y condiciones la que la situación actual de confinamiento obligatorio y cierre de las aulas en todo el país ha puesto de manifiesto, en su máxima expresión.

Desde hace años, de hecho, la Sociología de la Educación ha señalado que las funciones de socialización y selección que llevan a cabo los sistemas educativos no actúan para todos los grupos sociales del mismo modo y que es precisamente su mayor o menor distancia con la cultura escolar (Bourdieu y Passeron, 1970) lo que explica los procesos de éxito, fracaso y/o abandono escolar (Tarabini, 2018). Mientras para unos aquello que se transmite en la escuela (tanto a nivel cognitivo como moral, en palabras de Parsons; a nivel instrumental y expresivo, como diría Bernstein) coincide, es reconocido, respetado y amplificado por lo que ocurre en sus entornos familiares y extraescolares, para otros supone un choque, una renuncia y/o una lucha constante. Mientras para unas hay continuidades, materiales y simbólicas, entre la escuela y la vida, para otras son dos mundos a parte, a menudo opuestos. Porque el conocimiento y las disposiciones escolares no son neutrales, ni universales; no existen como hechos descontextualizados. Tienen una naturaleza histórica y social y, como tales, están mediadas por las relaciones de poder que atraviesan cualquier forma de sociedad (Young, 1971). De este modo, clase, género y etnicidad -como principales, aunque no únicos, ejes de desigualdad-cruzan de forma transversal tanto la selección, organización y transmisión del conocimiento escolar como su adquisición; median las diferentes formas de experiencia escolar; intervienen en las formas de ejercer de alumno/a y de docente; articulan las relaciones pedagógicas en toda su amplitud.

La situación actual de confinamiento, amplia y refuerza estas formas de desigualdad. Amplifica la desigualdad de condiciones de familias, alumnado y profesorado para hacer de la educación una herramienta de emancipación social. Dichas desigualdades, sin embargo, no se explican única ni exclusivamente por una brecha digital -como argumentaré a continuación- sino por una brecha de sentido; por una brecha de condiciones para que la escuela pueda ejercer su rol de transmisión de conocimientos y competencias profundas y relevantes para todo el alumnado.

\section{La evidencia de las brechas: las múltiples caras de la desigualdad}

Como decía, el contexto de confinamiento ha puesto de manifiesto las múltiples brechas que atraviesan nuestro sistema educativo y que sitúan sistemáticamente a unos colectivos sociales en situación de mayor desventaja escolar. Son numerosos los artículos de prensa e informes especializados que se han publicado recientemente sobre esta cuestión ${ }^{3}$. Brechas económicas, sociales, culturales, emocionales, digitales que el confinamiento evidencia en sus formas más crudas. Jóvenes solos/as, desorientados/as, angustiados/ as. Familias que se quedan sin trabajo. Condiciones de habilidad tremendamente dispares. Desigualdades

3 A modo de ejemplos puede consultarse: Calderón (2020) o Bonal y González (2020). 
cruciales en las posibilidades de desarrollar actividades educativas con los hijos/as. Falta de acceso a recursos tecnológicos, escasa competencia digital y un largo etcétera.

Estas brechas no solo se han evidenciado desde el punto de vista familiar, sino también desde el punto de vista escolar. En un extremo, centros que casi 'ni han notado' el paso de la escuela presencial a la virtual; en el otro, docentes desbordados, abrumados, superados por un entorno digital que les es totalmente ajeno. En un lado, centros que casi desde el primer día de confinamiento mandan actividades educativas obligatorias y evaluables a su alumnado y que han convertido los espacios familiares prácticamente en entornos escolares; en el otro, centros que luchan desesperadamente por encontrar a aquel alumnado con el que no han podido volver a contactar desde el primer día de confinamiento. Unos preocupados porque su alumnado no pierda el 'ritmo' de aprendizaje y adquisición de competencias. Otros porque sus alumnos/as puedan alimentarse en buenas condiciones ahora que los comedores escolares se han paralizado. Unos centrados en el rendimiento académico. Otros en el acompañamiento emocional de niños, niñas y jóvenes que viven la situación actual desde la soledad, la angustia y el miedo; por lo que viene y lo que vendrá. Por la pérdida de futuro y de predictibilidad.

Y si bien es cierto que entre estos extremos hay múltiples puntos intermedios, que no todo es blanco o negro y que se pueden combinar objetivos y preocupaciones también lo es que esta polarización está marcada por un elemento central: la clase social. Esta es la clave para explicar la desigualdad de condiciones que experimentan familias, alumnado y centros educativos para abordar la situación actual. Una clase social que ya existía antes del confinamiento y que ahora se evidencia y se refuerza. Una clase social que no solo se expresa en aspectos económico-materiales, sino también en dimensiones culturales y emocionales. Una clase social que atraviesa familias, centros, relaciones, experiencias vitales y posibilidades de aprendizaje eminentemente desiguales en este contexto de confinamiento generalizado.

Porque a pesar de que la situación de pandemia global genera incertidumbres y miedos de forma generalizada, también lo es, como explica Boaventura de Sousa Santos (2009), que la incertidumbre, el miedo y la esperanza no están igualmente distribuidos entre todos los grupos sociales. El riesgo de precariedad, de expulsión, de violencia o de enfermedad, también de muerte argumenta el autor, nunca han actuado para todos por igual. Ahora tampoco. Y no se trata solo de disponer o no de dispositivos tecnológicos para poder realizar las actividades escolares que mandan los centros educativos. Se trata también de disponer de acompañamiento y ayuda para realizar dichas actividades. Se trata de preguntarse si se puede educar con miedo, con falta de esperanza, con falta de futuro. Porque la desigualdad social, antes y durante el confinamiento, se expresa sobre todo a partir del capital cultural de las familias; de su mayor o menor distancia -como he argumentado en el apartado anterior- con las normas, requisitos, conocimientos, dados por supuesto de la institución escolar. Por ello, como afirma Martín Criado (2020), delegar la responsabilidad de la instrucción a las familias agudiza -ahora y siempre- las desigualdades sociales.

La desigualdad se expresa también en forma de capital emocional. Un capital mediado por el género y la clase social (Reay, 2004) y que nada tiene que ver con la mayor o menor preocupación de las familias de diferente clase social por la situación educativa de sus hijos e hijas. La evidencia empírica se ha encargado de desmentir reiteradamente el supuesto según el cual son las familias de clase media profesional las que más se preocupan por la educación de sus hijos e hijas (Lareau, 2003). Dichas familias no tienen más preocupación, lo que tienen son más estrategias, más legitimidad, más voz y más recursos para mostrar dicha preocupación y ejercerla a su favor. Es en este marco en el que debe entenderse el concepto de capital 
emocional. Porque la angustia, el miedo, la sensación de incapacidad para hacer frente a los requisitos de la institución escolar están claramente marcados por la clase social. Desde siempre y ahora, en la distancia y frente al desconcierto, aún más.

Por ello, y para evitar que la situación actual agrave aún más la desigualdad social, hacen falta políticas que actúen sobre los diferentes focos generadores y reproductores de injusticia. Políticas de redistribución que garanticen la igualdad de recursos (económicos, culturales, tecnológicos, etc.) entre familias y escuelas. Políticas de reconocimiento que asuman que la pandemia global y el confinamiento obligado no afectan por igual a todos los contextos escolares y familiares y que, por tanto, sean capaces de compensar la desigualdad. Y políticas de cuidado, que pongan el acompañamiento y la escucha en el centro de acción. Porque sabemos que cuando los y las jóvenes abandonan la escuela lo hacen desde una profunda desvinculación con aquello escolar (Tarabini et al., 2015). Desvinculación que pasa tanto por lo cognitivo como por lo conductual y, sobre todo, por lo emocional. Desvinculación ahora también física y tecnológica, pero que no llega sola ni se crea en medio de la nada.

Es en este contexto en el que, como decía, cobra pleno sentido preguntarse por las funciones de la escuela. ¿Pueden llevarse a cabo desde la distancia? ¿En qué condiciones? ¿Cómo se redibujan y resignifican? Como he argumentado, la situación actual aumenta la desigualdad educativa y, por tanto, limita el rol de la escuela como institución garante de la igualdad social. Pero ello no se explica única ni prioritariamente por las enormes diferencias que existen entre familias y centros para ejercer su función educativa y que la situación actual evidencia y amplifica. Ello se explica, a mi entender, por una crisis de sentido de la institución escolar que actúa de forma especialmente acusada en los contextos sociales más vulnerables y que la situación actual, de nuevo, evidencia, amplifica y magnifica. ¿Quién puede seguir aprendiendo cuando a la distancia cultural con la escuela y a la distancia afectiva con los docentes se le añade ahora una distancia física? ¿Quién puede concentrarse en realizar las tareas escolares cuando está confinado en un espacio compartido de 20 metros cuadrados sin luz natural? ¿Cómo se puede crear comunidad escolar cuando es imposible conectarse virtualmente con todo el alumnado que forma el grupo clase? ¿Cómo se puede capacitar para aprender y vivir en sociedad sin reconocer y abordar la profunda disparidad de condiciones desde las cuáles se está viviendo la situación actual?

Es aquí donde se pone de manifiesto la absoluta necesidad de la escuela, como institución social, para los grupos sociales más vulnerables. La escuela, como espacio físico y también como espacio simbólico, puede y debe ofrecer la posibilidad de ir más allá de los marcos de lo pensable y de lo imaginable. Debe ser un espacio de protección física, social y emocional para niños, niñas y jóvenes. De ahí viene su sentido. Un sentido que solo adquirirá su pleno valor si garantiza la adquisición de conocimientos profundos y relevantes para todos los estudiantes. Si actúa como espacio de inclusión -en sentido amplio- de todo el alumnado.

\section{El sentido de la escuela: un aprendizaje profundo para todo el alumnado}

Edgar Morin (1999) se refiere a cuatro principios de la educación pertinente: la contextualización, la globalización, la multidimensionalidad y la complejidad. Contextualizar el conocimiento escolar, por una parte, se refiere a la importancia de considerar el contexto social, económico, cultural y personal en que se inscribe el conocimiento educativo. Este contexto, de hecho, es fundamental para dotarlo de sentido. En la situación actual es impredecible preguntarse qué contexto de recepción del conocimiento escolar 
tienen niños, niñas y jóvenes de diferentes grupos sociales. De qué manera el confinamiento afecta a las posibilidades, materiales culturales, emocionales de adquirir, realizar e interiorizar dicho conocimiento. Pero estas preguntas no son propias de la situación actual. La contextualización del conocimiento escolar es fundamental para que este sea pertinente en cualquier situación y época histórica y su falta de contextualización es precisamente lo que lleva a muchos y muchas jóvenes a sentir que la escuela 'no es para ellos' (Tarabini, 2018).

Y lo mismo ocurre con la globalización, la multidimensionalidad o la complejidad del conocimiento escolar. Para aprender y para que este aprendizaje sea profundo y significativo es fundamental conectar las partes con el todo, lo local con lo global, la experiencia cotidiana concreta con situaciones más amplias, generales y universales. Como afirma Young (2013), el conocimiento poderoso, aquel que abre posibilidades de emancipación social, es el que habilita para pensar más allá de las realidades inmediatas, el que puede transferirse de un contexto a otro, el que adquiere relevancia más allá de su aplicabilidad inmediata. Es aquel capaz de dar sentido a la realidad cotidiana a partir de marcos de significado globales y que, a su vez, refuerza y matiza los marcos teóricos existentes a partir del significado que adquieren en contextos particulares. Las preguntas que debemos formularnos, pues, para abordar el sentido de la institución escolar en la actualidad son las siguientes: ¿Cuáles son las condiciones materiales, pedagógicas, sociales y profesionales para garantizar el acceso y adquisición de todo el alumnado a este tipo de conocimiento? ¿Qué elementos internos y externos a la institución escolar habilitan al alumnado para aprender?

Desde mi punto de vista, y siguiendo la propuesta de Dubet (2010), uno de los elementos centrales para entender el declive de la institución escolar es la crisis del propio proceso de socialización y transmisión cultural. Una crisis, que de forma contraria a lo que a menudo se cree, no solo responde a cambios externos o exógenos a la escuela. También se explica por factores endógenos vinculados con tres aspectos centrales que han caracterizado históricamente a la escuela como institución social: el supuesto de una cultura universal; la legitimidad de la figura docente; y una supuesta escisión entre el mundo de la escuela y el mundo exterior. Como explica el autor, y centrándome ahora en el primer aspecto, los procesos de democratización escolar han conllevado una mayor heterogeneidad social que, a su vez, han erosionado la identificación mecánica por parte de familias y alumnado con los valores homogéneos y los conocimientos aparentemente universales de la escuela. Se ha puesto de manifiesto la arbitrariedad cultural que representa la escuela y por ello ha entrado en declive. Porque en una escuela heterogénea socialmente la pertinencia del conocimiento escolar no puede darse por descontado y los procesos de contextualización del conocimiento adquieren más complejidad. En este mismo sentido Menéndez (cit. por Varela, 2020), afirma que la crisis de la escuela está vinculada con su propia organización, todavía industrial, donde todo el alumnado se tienen que adaptar y tiene que recibir lo mismo al mismo tiempo.

Es más, desde mi punto de vista, hay dos elementos fundamentales para que la función de la escuela, tal como la hemos definido, se pueda desarrollar: por una parte, la importancia de la presencia física como condición necesaria para garantizar la interacción plena necesaria en los procesos de transmisión cultural; por otra, el rol del docente como acompañante en los procesos de aprendizaje y desarrollo personal. Veámoslo.

Por una parte, y partiendo de la naturaleza multidimensional del aprendizaje, es imprescindible tener en cuenta que éste no solo se genera de forma individual y que, además, no se genera solo con y desde la cabeza. Los seres humanos no somos solo seres cognitivos, somos también seres sociales, emociona- 
les, relacionales. Por ello, la educación es profundamente un acto social. Y por ello también cognición y emoción no se pueden separar. Como tampoco se pueden separar tantas otras dicotomías que a menudo a traviesan el mundo escolar: teoría y práctica, sujeto y objeto, alma y cuerpo. ¿Cómo puede un/a alumno/a conectar lo cognitivo si lo emocional está roto? ¿Como puede activar lo emocional si lo social está ausente? ¿Cómo puede aprender sin sentir y sin experimentar?

En este punto es fundamental revindicar el rol de la escuela presencial como condición necesaria -aunque no suficiente- para posibilitar la adquisición de conocimientos profundos y relevantes para todos y todas las estudiantes. Ciertamente, los entornos presenciales y virtuales son menos dicotómicos de lo que los discursos alarmistas y mediáticos a menudo transmiten. Ni estar físicamente en la escuela implica necesariamente escucha, atención y colaboración, ni estar en el mundo virtual tiene por qué asociarse con procesos de individualización o de aislamiento social. Hay numerosos ejemplos del uso de tecnologías digitales como fuente de intercambio, cooperación y reflexión. Asimismo, la distancia física que implica el confinamiento no tiene por qué convertirse forzosamente en distancia emocional. O dicho de otro modo, la distancia física es una forma más de distancia que puede actuar de forma independiente o interconectada con otras formas de distancia -cultural, afectiva, social- entre familias, jóvenes y escuelas.

Ahora bien, hay elementos claves de la experiencia escolar que pasan por la vivencia física, presencial, corporal, sensorial del espacio escolar; de sus actores, de sus sensaciones, de sus olores, de sus colores ${ }^{4}$. Sin duda, en el mundo virtual también se siente. Pero no se toca. Porque hay formas de interacción que requieren de la presencia, del contacto físico, del movimiento, para poderse llevar a cabo y que el mundo virtual, por su naturaleza y características no permite. En este sentido, aprender en entornos virtuales nunca puede conseguirse de forma plena sin la interacción con espacios presenciales.

Ahora bien, la presencia por sí misma no es necesariamente placentera, ni liberadora o emancipadora. Profundizar en este argumento me alejaría del objetivo central de este texto, pero tenemos suficientes evidencias de que la presencia escolar está demasiado a menudo orientada a docilizar los cuerpos, a disciplinar las mentes, a escindir alma y razón (Dubet, 2010; Youdell, 2006). Para recuperar el sentido de la escuela es, por tanto, necesario también repensar el sentido de las presencias escolares. Porque no se puede escindir el mundo de la escuela del mundo exterior, como no se pueden escindir las diferentes partes del ser.

Por otra parte, es imprescindible tener en cuenta el rol de los y las docentes para que los conocimientos escolares sean profundos y las experiencias escolares significativas. De hecho, para garantizar la relevancia del conocimiento escolar es fundamental atender a su forma de transmisión (pedagogía) y no solo a su definición y selección (currículum). Coincido con Young (2010) en la necesidad de diferenciar conceptualmente entre currículum y pedagogía. Es decir, a pesar de que el currículum, entendido como aquel conocimiento que se considera importante que adquiera todo el alumnado, pueda ser pertinente y válido por sí mismo, dicha pertinencia puede verse cuestionada a través de la propia relación pedagógica, a través de la distancia física, cultural, emocional y simbólica entre profesorado y alumnado. Es por ello que para recuperar el sentido de la institución escolar es imprescindible replantearse las fuentes de legitimidad de la figura docente. Porque la autoridad pedagógica, hoy en día, no puede darse por descontado. Debe 'ganarse' en el día a día de la interacción escolar.

4 Quiero agradecer a Judith Jacovkis del GEPS-UAB y a Núria Valles de STS-b por las reflexiones (virtuales) que hemos compartido sobre el valor de la presencia física en las formas de interacción social. 
Preguntarse por el rol del y la docente en el marco de la institución escolar actual implica también reconocer que la labor docente, como todas las tareas basadas en las relaciones humanas, conlleva un fuerte trabajo emocional (Hargreaves, 2001). Tal como afirma Freire (1972, citado en Lynch y Baker, 2005:150), 'los buenos docentes quieren a sus estudiantes', en el sentido de estar profundamente implicados en su desarrollo de forma que les haga libres. En este sentido, siguiendo la propuesta de Hargreaves (2003), es imprescindible que las escuelas sean tanto comunidades de aprendizaje como comunidades de cuidado. Porque aprender y cuidar van profundamente cogidos de la mano. Porque si bien la escuela no puede renunciar a enseñar, es evidente que no se puede enseñar sin cuidar. Por ello es fundamental reclamar una figura docente capaz de acompañar a su alumnado en el sentido más amplio de la palabra. Acompañar significa estar o ir en compañía del otro, existir junto al otro, participar en sus sentimientos. Y este es el rol que deben realizar los y las docentes para que la función de la escuela de formación del sujeto y transmisión cultural pueda llevarse a cabo. Se trata, en definitiva, de alejarse de lo que Lingard y Keddie (2013) definen como pedagogías de la indiferencia, aquellas que producen y/o legitiman la desigualdad social.

\section{A modo de conclusión}

El objetivo de este artículo ha sido reflexionar sobre el sentido de la institución escolar en una situación inédita de pandemia global generada por el COVID-19 y confinamiento obligatorio de la población. La paralización de la actividad presencial de las escuelas ha puesto en evidencia las amplias brechas que existen entre familias y escuelas para desarrollar su función educativa y ha evidenciado las múltiples formas de exclusión que limitan la función igualadora de debería cumplir la institución escolar. He argumentado, de hecho, que la función de la escuela pública, común, obligatoria y gratuita como instrumento de igualación social es precisamente lo que le confiere su especificidad en tanto que institución social. Una especificidad que implica garantizar la transmisión y adquisición de conocimientos profundos y relevantes para todos y todas las estudiantes y que se ve profundamente amenazada por las formas de segregación, desvinculación y expulsión que atraviesan nuestro sistema educativo.

Este riesgo, sin embargo, no se genera única, exclusiva o prioritariamente por la brecha digital que ha generado la situación actual. Se genera, como he argumentado, por una crisis de sentido de la institución escolar que se explica por la falta de profundidad y relevancia de los conocimientos escolares, especialmente para los colectivos sociales más vulnerables. Y esta falta de relevancia es lo que lleva a los grupos sociales de menor estatus socioeconómico y cultural a ocupar de forma abrumadoramente mayoritaria las estadísticas de fracaso y abandono escolar. Porque, evidentemente, la escuela es la base de la movilidad social de numerosas personas de clase trabajadora a título individual, pero a nivel colectivo el sistema educativo no contribuye a la emancipación social de los grupos sociales más desfavorecidos. Y de ahí viene su crisis de sentido. Porque una escuela que expulsa, física y simbólicamente, de espacios, de aprendizajes, de respecto y de experiencias satisfactorias a tantos y tantas jóvenes es una escuela que pierde su función principal. Es una escuela vacía.

He argumentado también que para garantizar la función de la escuela como institución de igualación social hacen falta dos condiciones imprescindibles: por un lado, la presencia y el contacto físico como forma para garantizar las formas de interacción profunda que requieren los procesos de transmisión cultural. Esta presencia, fuera del espacio familiar, es también un elemento crucial para 'llevar' a los alumnos más allá de su experiencia cotidiana y abrirles nuevos mundos y ventanas de oportunidad. Por otro lado, he defendido la figura del y la docente como acompañante para garantizar que las escuelas actúen simul- 
táneamente como comunidades de aprendizaje y de cuidado. Porque enseñar y cuidar, aprender y sentirse cuidado/a son dos caras de la misma moneda.

Sin duda, sería injusto presuponer que las escuelas y/o los docentes a nivel individual son los principales responsables de esta crisis de sentido. Escuelas y docentes deben asumir su responsabilidad, como actores críticos y reflexivos (Perrenoud, 2001), en el marco de un contexto más amplio marcado por políticas educativas que les condicionan, constriñen y/o habilitan. Asimismo, sería naif imaginar que la escuela per sí misma puede superar todas las desigualdades generadas fuera del espacio escolar. Las sociedades capitalistas, en diferente grado, siempre producen desigualdades a nivel económico, laboral, sanitario, habitacional que van más allá de la intervención estrictamente escolar. Ahora bien, la escuela como institución social debe actuar como espacio de protección y garantía de derechos para toda la infancia y la juventud y eso implica garantizar igualdad de condiciones para que todos los centros y todas las familias puedan ejercer su función educativa. Una igualdad de condiciones que pasa por políticas de redistribución, reconocimiento y cuidado entre y dentro de los centros educativos y que si bien no depende solo de escuelas y docentes a título individual, les necesita como sus principales aliados.

\section{Referencias bibliográficas}

Althusser, Louis (1985): "El aparato ideológico del estado escolar como aparato dominante" en Alain Gras (Ed.): Sociología de la educación. Textos fundamentales. Madrid: Narcea.

Bernstein, Basil (1985a): “Clases sociales, lenguaje y socialización”. Revista Colombiana de Educación, 5.

Bernstein, Basil (1985b): “Clasificación y enmarcación del conocimiento educativo”. Revista Colombiana de Educación, 15.

Bonal, Xavier y González, Sheila (2020): “Confinamiento y efecto escuela”. elPeriódico.com. https://www. elperiodico.com/es/opinion/20200406/efecto-coronavirus-desigualdad-escuelas-xavier-bonal-sheila-gonzalez-7919442, publicado el 6 de abril de 2020.

Bonal, Xavier (1998). Sociología de la Educación: una aproximación crítica a las corrientes contemporaneas. Barcelona: Paidós

Bourdieu, Pierre y Passeron, Jean-Claude (1970). La Reproduction. Éléments pour une théorie du système d'enseignement. Paris: les editions de minuit.

Bowles, Samuel y Gintis, Herbert (1976). Schooling in Capitalist America. London: Routledge and Kegan Paul.

Calderón, Daniel (2020). Jóvenes y desigualdad digital: las brechas de acceso, competencias y uso. Madrid: Centro reina Sofía sobre adolescencia y juventud.

De Sousa Santos, Boaventura (2016): "La incertidumbre: entre el miedo y la esperanza” en Boaventura de Sousa Santos. La dificil democracia. Una mirada desde la periferia europea. Madrid: Akal.

Dubet, François (2010): “Crisis de transmisión y declive de la institución”. Política y Sociedad, 47 (2), 15-25.

Gimeno Sacristán, José (2000). La educación obligatoria: su sentido educativo y social. Madrid: Morata. 
Hargreaves, Andy (2003). Teaching in the Knowledge Society. Education in the Age of Insecurity. New York and London: Teachers College Press.

Hargreaves, Andy (2001): “Emotional Geographies of Teaching”. Teachers College Record, 103 (6), 10561080.

Lareau, Annette (2003). Unequal Childhoods. Class, race and family life. Berkeley: University of California Press.

Lingard, Bob y Keddie, Amanda (2013): "Redistribution, Recognition and Representation: Working Against Pedagogies of Indifference”. Pedagogy, Culture \& Society, 21 (3), 427-447.

Lynch, Kathleen y Baker, John (2005): "Equality in education An equality of condition perspective". Theory and Research in Education, 3 (2), 131-164.

Martín Criado, Enrique (2000): “El confinamiento aumenta la desigualdad educativa (y no es culpa de los padres)". Entramados sociales, web de divulgación sociológica. Acceso: https://entramadossociales.org/ educacion/el-confinamiento-aumenta-la-desigualdad-educativa-y-no-es-culpa-de-los-padres/, publicado el 1 de abril de 2020 .

Morin, Edgar (1999). Los siete saberes necesarios para la educación del futuro. París: UNESCO.

Parsons, Talcott (1990): "El aula como sistema social: Algunas de sus funciones en la sociedad americana”. Educación y Sociedad, 6.

Perrenoud, Philippe (2011). Desarrollar la Práctica Reflexiva en el Oficio de Enseñar. Barcelona: Graó.

Reay, Diane (2010): “Identity Making in Schools and Classrooms". en Margaret Wetherell y Chandra Talpade Mohanty (Eds). The Sage Handbook of Identities. Londre: Sage.

Reay, Diane (2004): “Gendering Bourdieu’s Concepts of Capitals? Emotional Capital, Women and Social class”. The Sociological Review, 52, 57-74.

Tarabini, Aina (2018). La escuela no es para ti. El rol de los centros educativos en el abandono escolar. Madrid: Octaedro.

Tarabini, Aina; Jacovkis, Judith y Montes Alejandro (2017). Los factores de la exclusión educativa en España: Mecanismos, perfiles y espacios de intervención. Madrid: UNICEF.

Tarabini, Aina; Curran, Marta; Montes, Alejandro, Parcerisa, LLuís (2015): “La vinculación escolar como antídoto del abandono escolar prematuro. Explorando el papel del habitus institucional”. Profesorado. Revista de currículum y formación de profesorado, 19 (3).

Youdell, Deborah (2006). Impossible Bodies, Impossible Selves: Exclusions and Student Subjectivities. Dordrecht: Springer.

Young, Michael (2013): "Powerful Knowledge: An Analytically Useful Concept or Just a 'Sexy Sounding Term’? A Response to John Beck's 'Powerful Knowledge, Esoteric Knowledge, Curriculum Knowledge"'. Cambridge Journal of Education, 43 (2), 195-198. 
Young, Michael (2010): “The Future of Education in a Knowledge Society: The Radical Case for a Subject-Based Curriculum”. Pacific-Asian Education, a Journal about Education in Pacific Circle countries, 22 (1), 21-32.

Young, Michael (Ed.) (1971). Knowledge and Control: New Directions for the Sociology of Education. London: Collier-Macmillan Publishers.

Varela, Alejandra (2020): “Coronavirus y educación. Pepe Menéndez. Antes ya teníamos una crisis de la escuela". Clarín. Acceso: https://www.clarin.com/revista-enie/ideas/pepe-menendez-crisis-escuela_0_gegH7q4NW.html, publicada el 10 de abril de 2020.

\section{Nota biográfica}

Aina Tarabini es Doctora en Sociología por la Universidad Autónoma de Barcelona, profesora agregada de la misma universidad e investigadora del grupo de investigación Globalización, Educación y Políticas Sociales (GEPS). Su investigación se desarrolla en el campo de la sociología de la educación y se centra en el análisis las desigualdades educativas. Está particularmente interesada en el estudio de las disposiciones, elecciones y transiciones educativas de los y las jóvenes, así como en el análisis de las prácticas pedagógicas desde la perspectiva de la justicia social. Actualmente es la investigadora principal del proyecto «Edupost16. La construcción de oportunidades educativas post 16. Un análisis de las transiciones educativas postobligatorias en entornos urbanos» (http://www.edupost16.es).

Orcid: https://orcid.org/0000-0002-6096-2450. 